\title{
Disease surveillance, capacity building and implementation of the International Health Regulations (IHR[2005])
}

Rebecca L Katz ${ }^{* 1,2}$, Jose A Fernandez ${ }^{3}$ and Scott JN McNabb4

\section{Introduction}

At the December 2009 Meeting of States Parties of the Biological Weapons Convention (BWC), U.S. Under Secretary of State Ellen Tauscher committed the U.S. Government (USG) to engaging the global community to achieving and sustaining the capabilities to combat infectious diseases and protect against biological threats. Specifically, she committed the USG to a series of actions, including international meetings on global disease surveillance and implementation of the International Health Regulations (IHR[2005]), designed to enhance global cooperation and provide momentum for sustained progress in this critical area. This journal supplement includes articles that capture key presentations from two meetings: the June 2010 workshop on Comprehensive Global Disease Surveillance held in Washington, D.C. and the August 2010 workshop on Implementation of the IHR(2005) held in Geneva, Switzerland. The supplement also highlights efforts underway to enhance disease surveillance and IHR(2005) implementation by global partners and frames the current USG efforts to enhance global cooperation in disease surveillance, capacity building, biothreat reduction, and IHR(2005) implementation.

The IHR(2005) provides a framework to promote global health security in the broadest sense. Public health emergencies of international concern (PHEICs), by definition, do not respect international boundaries, and the IHR(2005) articulates a vision of solidarity that a common vulnerability to microbial and other threats should elicit. A common interest exists for all countries to possess the capacities and capabilities identified in the IHR(2005) to detect, assess, report, and respond to public health threats, whether they are naturally occurring, accidental, or deliberate in origin. This interest is neither solely a public health interest, nor a security interest, but a

*Correspondence: KatzRL@state.gov

'U.S. Department of State, Washington, DC, USA

Full list of author information is available at the end of the article human interest. Accordingly, the public health and security communities have found it increasingly beneficial to work together to advance their shared objectives in this particular area. While these two communities operate in distinct spheres, there is an area where the public health and security spheres overlap. These workshops brought the two communities together to clarify the connections between these spheres and to promote and enhance cooperative efforts between them to advance IHR(2005) implementation internationally in an effective, meaningful, and sustainable manner.

\section{Working towards comprehensive global disease surveillance}

On June $16^{\text {th }}$ and $17^{\text {th }}, 2010$ more than 140 health and security experts from 30 countries gathered in Washington, D.C. to discuss the fundamental components of comprehensive disease surveillance, impediments to implementing efficient and effective systems, and lessons and recommendations under the IHR(2005) that help build core disease surveillance capacity. The meeting identified policy imperatives necessary to achieve functional, comprehensive systems, particularly in lowresource settings and provided a venue for funders and aid recipients to discuss the core capacities for surveillance, as outlined in Annex 1 of the IHR(2005).

The June workshop included presentations from U.S. senior officials from Department of State (DoS), Department of Health and Human Services (DHHS), and the Department of Defense (DoD). The U.S. National Security Staff highlighted the political-level commitment for increased coordination between the health and security communities. Representatives from across the USG described their agencies' efforts to build global disease surveillance capacity, and global experts gave overviews of essential components of effective surveillance; including human workforce development, communications, epidemiologic capacity, and the human/animal interface. The remainder of the workshop was spent in 
* The international community must continue efforts to bridge the gap between the security and the public health sectors to ensure the successful implementation of the IHR(2005). These communities share mutual goals, particularly as related to disease surveillance, yet often fail to communicate with each other effectively.

* There must be increased stakeholder involvement in IHR(2005) implementation; success requires inter-ministerial cooperation.

* While countries may require technical and financial assistance in IHR(2005) implementation, success is ultimately the responsibility of each individual country. Countries should work in collaboration with the WHO to request technical support as needed and continue to leverage the expertise and resources of the donor community. At the same time, each country must be responsible for setting its own goals and timelines, determining its needs, and conducting realistic assessments of progress and shortcomings.

* Sustainability is critical to successful IHR(2005) implementation in developing countries, especially in terms of human capital and laboratory capacity.

* The chemical, nuclear and radiological threats encompassed in IHR(2005) present a unique challenge to the surveillance and response community in both developed and developing countries. Surveillance networks typically reside in health ministries and focus predominately on infectious diseases. Surveillance capacity for public health incidents involving chemical, nuclear, or radiological material is underdeveloped or nonexistent in much of the world. New cooperative relationships between the public health community and the security community may be able to fill these gaps.

Figure 1. Key themes from breakout sessions at the Workshop on Moving Towards Comprehensive Global Disease Surveillance.

break-out sessions, enabling participants to share national viewpoints, experiences, and suggestions for cooperative efforts (see Figure 1).

In this journal supplement, we include six articles drawn directly from this workshop. Drs. McNabb and Chungong provide an overview of global surveillance elements, the important scientific, political, and technologic drivers of public health surveillance, and the surveillance core capacities required for compliance with the IHR(2005). Drs. Kant and Krishnan describe how information and communication technology is being used for disease surveillance in India. Mr. Johns and Dr. Blazes discuss how the Department of Defense is helping nations building core capacities for IHR(2005). Dr. Nsubuga from the Centers for Disease Control and Prevention (CDC), along with colleagues from the U.S. Agency for International Development (USAID), the Africa Field Epidemiology Network (AFENET) and CDC present mechanisms for strengthening surveillance and response capacity using the health systems strengthening agenda for developing countries. Dr. Andrus and colleagues from the Pan American Health Organization (PAHO) write about global health security in the context of the $\operatorname{IHR}(2005)$, with specific examples of how $\operatorname{IHR}(2005)$ guided the response to yellow fever in Paraguay and the $\mathrm{H} 1 \mathrm{~N} 1$ pandemic. Also in this supplement is the overview of the USG agencies and offices engaged in building global capacity for disease surveillance, as representatives presented it at this meeting.

\section{Implementation of the IHR(2005)}

On August 20 $0^{\text {th }}, 2010$ a follow-on workshop was held at the Palais des Nations in Geneva, Switzerland co-hosted by the BWC Implementation Support Unit. This workshop again brought more than 100 experts from around the world together for detailed discussion of lessons learned from national experiences implementing the IHR(2005) and regional efforts to support capacity building. The aim of this workshop was to share insights into the practical implementation of the $\operatorname{IHR}(2005)$, to identify and address obstacles, and to facilitate sustainable, long-term collaborations. Speakers representing four WHO regions delivered national presentations, including Uganda, represented in the article by Wamala, et al. WHO representatives spoke about international collaboration efforts necessary for IHR(2005) implementation and representatives from the AFENET and the American Society for Microbiology (ASM) spoke about capacity building efforts. These presentations are represented by articles by Dr. Specter and colleagues from ASM, and by Dr. Musenero and colleagues from AFENET.

Several major themes emerged from the meeting (see Figure 2), as well as specific challenges identified by 
* Successful implementation of IHR(2005) requires broad support within and beyond the health sector, and direct support from senior political levels.

* Effective implementation requires multi-sectoral engagement and active participation across ministries. Many countries struggle with engaging non-health sectors, particularly in non-emergency situations.

* Regions face diverse and unique needs; availability of resources varies within and across nations. In many regions, models for collaboration and cooperation are already in place that can and should be used as a platform for IHR(2005) implementation.

* Information sharing is essential, and internet-based information sharing should be encouraged.

* Metrics are needed to measure capacity and progress towards achieving IHR(2005) core capacities. These metrics may vary according to particular national conditions and resources.

Figure 2. Key themes, concerns and suggestions from participants at the Meeting on Implementation of the IHR(2005).

participants. Some of the specific challenges to successful IHR(2005) implementation include:

- Some countries struggle with gaps in resources, particularly human resources. Participants emphasized the importance of regional training centers to address workforce shortages and training gaps.

- Meeting IHR(2005) obligations at Points of Entry is a universal challenge, involving human resources and multi-sectoral engagement and communication.

- The safe and effective transportation of specimens and samples remains difficult in many parts of the world.

- There is a need for better laboratory infrastructure. Specifically, labs need broad spectrum diagnostics for rare diseases and common reagents.

- Some countries have had success in developing core capacities at the national level, but found it challenging to make substantial progress in developing capacity at the local level.

- Some countries are focused on building basic public health infrastructure to address endemic health needs, and must prioritize developing this basic infrastructure before focusing specifically on IHR(2005) compliance.

Workshop participants discussed a set of eight draft principles for capacity building and global cooperation for implementing $\operatorname{IHR}(2005)$. They include:

1. In today's interdependent and interconnected world, health security requires coordinated action and cooperation among members of the international community.

2. No single institution or country has all the capacities needed to effectively respond to international public health emergencies. An effective response to these events requires cooperation among multiple sectors and multiple partner countries, as well as the WHO.

3. The $\operatorname{IHR}(2005)$ provide a critical and universal framework for promoting global health security.
4. Early detection, rapid reporting, and effective response are critical to prevent or halt the international spread of disease.

5. Rapid and timely communications between countries and with the WHO is critical for the response to international public health emergencies.

6. Strong health systems are critical to each country's ability to prepare for and respond to both routine public health events and public health emergencies with international impact.

7. Capacity-building must be practical, sustainable, collaborative, and based on the needs of each country. In this regard, these efforts must contribute to the strengthening of each country's day-to-day capacities to detect and respond to public health events.

8. The development and maintenance of the $\operatorname{IHR}(2005)$ core capacities require a significant investment on the part of all countries. To maximize the effectiveness and efficiency of these capacity-building activities, it is important to take full advantage of opportunities for collaboration and coordination among partners.

\section{Additional policy issues}

Several active participants in the summer meetings have articles in this supplement relevant to IHR(2005), disease surveillance and capacity building. Dr. Bakanidze from Georgia and her co-authors write about biosafety and biosecurity as pillars of international health security, and discuss how Georgia is building a biosafety regime using the international guidelines provided by IHR(2005), the BWC and United Nations Security Council Resolution 1540. Dr. Sobers and her colleagues from Barbados detail the island nation's experience with $\mathrm{H} 1 \mathrm{~N} 1$ and the actions taken by the government to mitigate the consequences of the disease on their country. Finally, colleagues from the CDC the Defense Threat Reduction Agency (DTRA) 
collaborate on a paper that provides a systems approach to strengthening national surveillance and detection of events of public health importance.

\section{Conclusions}

Representing the desire to foster global collaboration and find both a common political and technical vision for full implementation of the IHR(2005), the representatives at the June and August meetings, as well as a growing network of international partners are achieving important consensus, activities, and outputs. Countries recognize gaps in disease surveillance capacity and needs for intra-country and inter-sector collaboration. They also face challenges in specific technical areas and in building leadership, communication, and collaboration. The platform for discussion and planning provided in June and August generated enthusiasm and targeted areas for intervention. The contributors to this supplement are codifying the vision for global disease surveillance and IHR(2005) implementation, and collectively, planning the future.

\section{Abbreviations}

AFENET, Africa Field Epidemiology Network; BWC, Biological Weapons Convention; CDC, Centers for Disease Control and Prevention; DHHS, Department of Health and Human Services; DoD, Department of Defense; DoS, Department of State; DTRA, Defense Threat Reduction Agency; IHR, International Health Regulations; PAHO, Pan American Health Organization; PHEIC, Public health emergency of international concern; USAID, United State Agency for International Development; USG, United States Government.

Competing interests

No competing interests to declare.
Authors' contributions

All authors contributed equally to the text.

\section{Acknowledgements}

We wish to thank our colleagues across the United States Government for their efforts to help plan the June and August 2010 meetings, especially Jennie Gromoll, Jennifer Bae, Jacqueline Smith, Lourdes Costacamps, Leana López, Leila Heintzelman, Jeremy Scherer and Will Chapman. We are also appreciative of the support from CRDF and M2K Solutions. We also thank all of the contributors from around the world who graciously participated in the workshops, including participants from the World Health Organization headquarters and regional offices. Finally, we are indebted to our colleagues across the U.S. Government, whose input and thoughts have been invaluable.

This article has been published as part of BMC Public Health Volume 10 Supplement 1, 2010: Disease surveillance, capacity building and implementation of the International Health Regulations [IHR(2005)] The full contents of the supplement are available online at http://www.biomedcentral.com/1471-2458/10?issue=S1.

\section{Disclaimer}

The views and opinions expressed in this editorial are expressly those of the contributing authors and not of the specific government agencies (Department of Health and Human Services and Department of State) or the United States Government.

\section{Author details}

'U.S. Department of State, Washington, DC, USA. ${ }^{2}$ George Washington University, School of Public Health and Health Services, Washington, DC, USA. ${ }^{3}$ U.S. Department of Health and Human Services, Washington, DC, USA. ${ }^{4}$ Emory University, Atlanta, GA, USA.

Published: 3 December 2010

doi:10.1186/1471-2458-10-S1-S1

Cite this article as: Katz RL, et al:: Disease surveillance, capacity building and implementation of the International Health Regulations (IHR[2005]). BMC

Public Health 2010, 10(Suppl 1):S1. 